\title{
Romiplostim Administration to a Preterm Neonate with Severe Prolonged Acquired Thrombocytopenia
}

Michael D. Kamitsuka, MD, Shrena Patel, MD, Richy T. Lee, MD, Robert D. Christensen, MD

\begin{abstract}
Platelet transfusions can be lifesaving for neonates with thrombocytopenic hemorrhage. However, multiple transfusions themselves convey risks and hazards. We cared for a preterm neonate with severe/prolonged acquired thrombocytopenia who received 61 platelet transfusions. Her platelet counts stabilized, and further transfusions were not needed, following three escalating doses of romiplostim.
\end{abstract}
Abbreviations
Tpo: thrombopoietin
DOL: day of life
NEC: necrotizing enterocolitis
$\mathrm{SQ}$ : subcutaneously
IPF: immature platelet fraction

Keywords: thrombopoietin; immature platelet fraction; platelet transfusion

\section{Established Facts}

- $\quad$ Thrombocytopenia is a common problem and may affect up to $70 \%$ of extremely low birth weight neonates

- Platelet transfusion is the current treatment option for neonates with severe symptomatic thrombocytopenia

- Mortality and risk for sepsis is increased in those requiring multiple platelet transfusions so other options need to be explored

Novel Insights

- Thrombopoietic stimulators like romiplostim may reduce the need for multiple platelet transfusions in neonates with symptomatic and persistent thrombocytopenia.

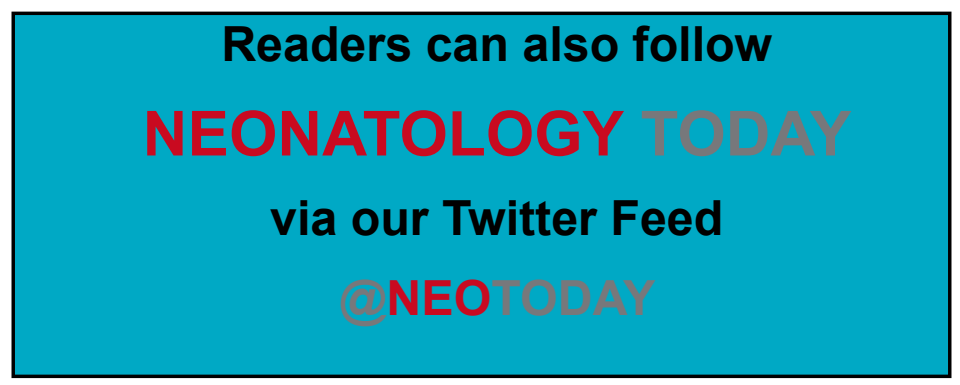

\section{Introduction}

The purpose of this report is to describe a case in which an extremely premature, low birth weight neonate, developed a prolonged course of thrombocytopenia requiring multiple platelet transfusions but subsequently stopped requiring platelet transfusions following a short course of the Tpo-mimetic, romiplostim.

In 1994 the principal physiological regulator of thrombopoiesis, thrombopoietin (Tpo), was cloned (1). Two recombinant forms were created: a full-length Tpo and a pegylated form containing only the receptor-binding domain. In early clinical trials, a few subjects receiving these molecules developed cross-reactive neutralizing antibodies against their endogenous Tpo, resulting in severe hypo-regenerative thrombocytopenia and aplastic anemia (2).

Second-generation Tpo-mimetics were developed, which do not share any sequence homology with endogenous Tpo, but stimulate thrombopoiesis by binding and activating the Tpo receptor (3). In 2018, the FDA approved romiplostim for use in children $>1$ year of age with immune thrombocytopenic purpura of $>6$ months (3). Although romiplostim has been used in cases of refractory thrombocytopenia in children, published use in neonates is limited (4). We would like to describe our experience of using romiplostim in a neonate with protracted thrombocytopenia.

\section{"The purpose of this report is to describe a case in which an extremely premature, low birth weight neonate, developed a prolonged course of thrombocytopenia requiring multiple platelet transfusions but subsequently stopped requiring platelet transfusions following a short course of the Tpo-mimetic, romiplostim."}

\section{Case Report}

A 540 gram, 23-week female was admitted to the neonatal intensive care unit with an initial platelet count of $201 \times 10^{3} / \mu \mathrm{L}$. The mother had been visiting Seattle from the East Coast when she delivered. Her prenatal laboratory values included non-reactive rapid plasma regain and human immunodeficiency virus titer, immune Rubella titer, normal cell-free DNA screen. Her platelet count was $151 \times 10^{3} / \mu \mathrm{L}$

On day of life (DOL) 10, the infant's abdomen became dusky and distended. No pneumatosis or free air was detected on her abdominal radiographs. Fluconazole was started after Candida albicans grew from a blood culture. Platelets were transfused four days later for a platelet count of $91 \times 10^{3} / \mu \mathrm{L}$; because she was a septic 23-week infant with a germinal matrix hemorrhage, we 
were trying to prevent further extension.

On DOL 30, her abdomen became distended and firm. Despite serial abdominal radiographs without pneumatosis or free air, necrotizing enterocolitis (NEC) was suspected. She was severely ill, requiring platelets and fresh frozen plasma transfusions for disseminated intravascular coagulation. Due to pancytopenia and extreme instability, exploratory surgery was deferred. During this time, she required 1-2 platelet transfusions daily to keep her platelet count $>100 \times 10^{3} / \mu \mathrm{L}$. Post transfusion platelet counts were rarely > $100 \times 10^{3} / \mu \mathrm{L}$ (Figure); therefore, starting on DOL 47, all aliquots were plasma reduced. Subsequent, immediate posttransfusion platelet counts were frequently $>200 \times 10^{3} / \mu \mathrm{L}$ but by 48 hours would invariably fall to $<100 \times 10^{3} / \mu \mathrm{L}$, as low as $9 \times 10^{3}$ I $\mu \mathrm{L}$.

Three weeks later, she was stable enough to go to surgery for a bowel obstruction. No intra-abdominal abscess, candidiasis, or necrotic bowel was identified. Her bowel was matted and friable. Handling the bowel trying to find the area of obstruction left her with multiple enterotomies. A diversion was not possible, so the abdomen was left open, and the baby was brought back to the NICU with the peritoneum open and the bowel exteriorized. She continued to require platelet transfusions, assumed to be consumptive related to her abdomen. The previous work-up for other possible causes for thrombocytopenia were negative, including urine polymerase chain reaction testing for cytomegalovirus and heparin-induced antibody testing (done due to the prolonged presence of a central line infusing heparinized solution). Laboratory values for liver failure, including coagulation factors, liver function tests, urine organic acids, and serum amino acid screen, were normal. Mother had a normal platelet count. The infant's platelet count was $>104 \times 10^{3} / \mu \mathrm{L}$ for the 10 first days of life, making alloimmune or autoimmune thrombocytopenia less likely. Her platelet count did not fall until she became septic.

Her clinical condition improved following her surgery, such that by DOL 87, she was transfused only for platelet counts $<50 \times 10^{3} /$ $\mu \mathrm{L}$. She returned to surgery eight weeks later, where a primary end-to-end anastomosis was performed. The bowel, but not the liver, could be reduced into the peritoneal cavity. The closure was accomplished with a vicryl mesh.

She received 61 platelet transfusions from DOL 14 to 145. The last three weeks of her hospital stay showing the platelet count in relation to platelet transfusions, dexamethasone start, and romiplostim are seen in the table. Dexamethasone was started for worsening bronchopulmonary dysplasia. Her last transfusion for a platelet count of $20 \times 10^{3} / \mu \mathrm{L}$ was the day after the initial romiplostim. Before her first dose of romiplostim (2 mcg/ $\mathrm{kg} / \mathrm{dose}$ ) subcutaneously (SQ), her immature platelet fraction (IPF) was $3.2 \%$ (NL 1.1-7.1\%). The platelet count continued to decrease, so a second dose (4 mcg/kg/dose) SQ was given after one week. The platelet count nadir was two days later. The first rise in the platelet count was four days after the second dose. After receiving her $3^{\text {rd }}$ dose, her IPF was $11.6 \%$. The mean platelet volume was $10.2 \mathrm{fL}$ with a platelet count of $70 \times 10^{3} / \mu \mathrm{L}$ One day after her third dose, she was transferred across the country to a NICU closer to her mother's home. A follow-up call to the hospital caring for this infant reported the platelet count was $240 \times 10^{3} / \mu \mathrm{L}$ on DOL 200 , and she had not received any platelet transfusions follow her transfer. No complications that we could attribute to romiplostim occurred prior to her transfer, and we did not have access to follow-up data to evaluate for complications after the transfer.

\section{Discussion}

This was an unusual case of chronic thrombocytopenia associated with a platelet count as low as $22 \times 10^{3} / \mu \mathrm{L}$ from DOL32 to as

\begin{tabular}{|c|c|c|c|c|}
\hline $\mathrm{DOL}$ & Platelet $\left(\times 10^{3} / \mu \mathrm{L}\right)$ & transfusion & medication & Dose $(\mathrm{mcg} / \mathrm{kg})$ \\
\hline 136 & 18 & yes & & \\
\hline 140 & 70 & & & \\
\hline 141 & & & dexamethasone & \\
\hline 142 & 22 & yes & & \\
\hline 143 & 85 & & & \\
\hline 144 & 60 & & Romiplostim & 2.4 \\
\hline 145 & 20 & yes & & \\
\hline 146 & 239 & & & \\
\hline 148 & 126 & & & \\
\hline 150 & 106 & & & \\
\hline 151 & & & Romiplostim & 4 \\
\hline 152 & 94 & & & \\
\hline 153 & 84 & & & \\
\hline 155 & 119 & & & \\
\hline 156 & 127 & & & \\
\hline 158 & & & Romiplostim & 4 \\
\hline 159 & 139 & & & \\
\hline 200 & 240 & & & \\
\hline
\end{tabular}




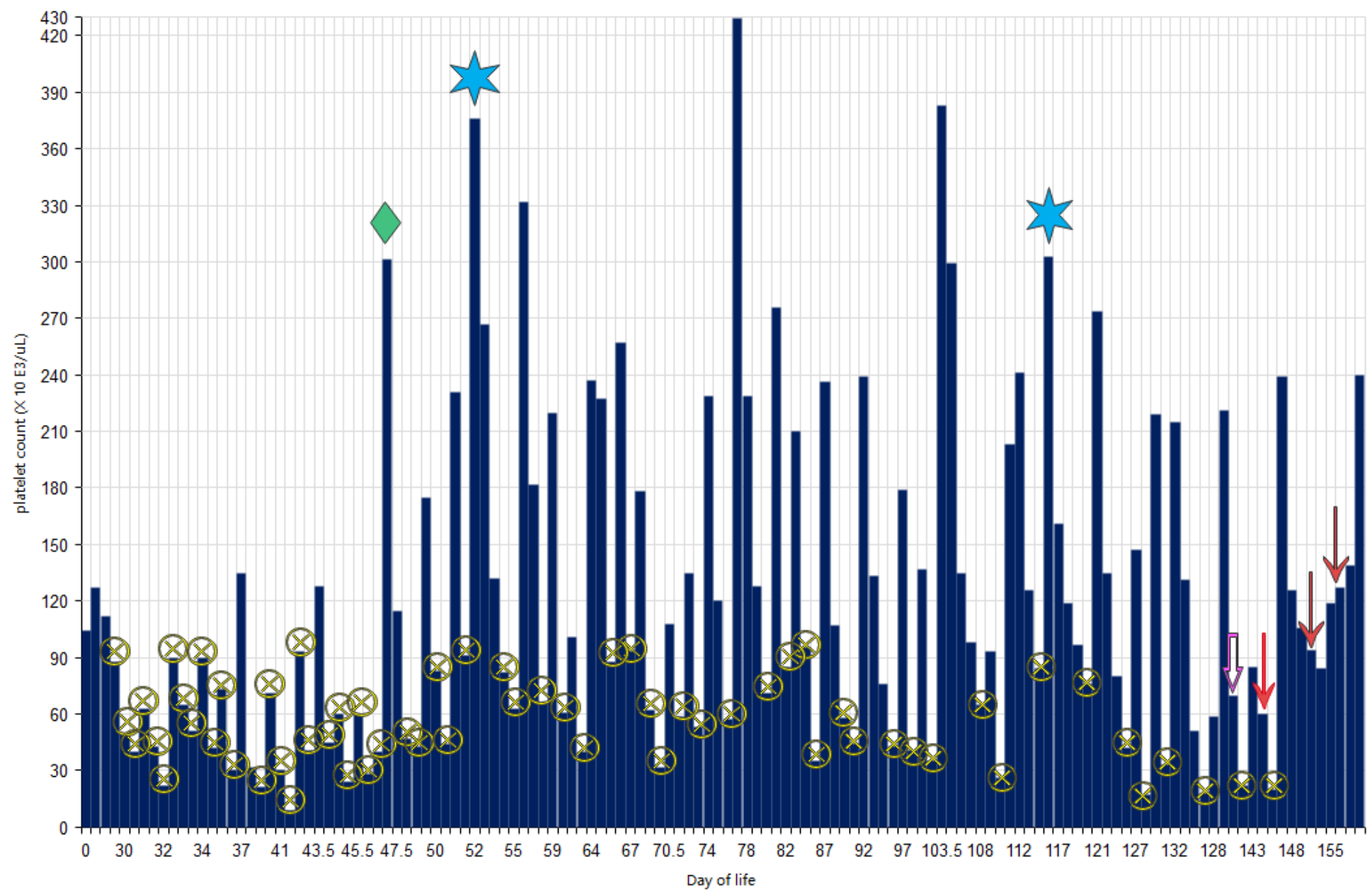

FIGURE

Shows platelet count in response to platelet transfusion and romiplostim

$\downarrow$ Romiplostim; \& platelet transfusion;

late as DOL 145. Following three doses of romiplostim, the baby never received another platelet transfusion.

\section{"In a survey of United States and Canadian neonatologists, Josephson found $30 \%-45 \%$ of the neonatologists would transfuse platelets in a sick preterm infants with platelet counts of $>50 \times 103 / \mu L$ (5), a threshold of $<20 \times$ $103 / \mu L$ was selected by $<5 \%$; and $50 \%$ selected $50 \times 103 / \mu L$ as the "transfusion" trigger for extremely low-birthweight neonates despite the absence of apparent bleeding (5)."}

plasma reduced platelets; $*$ surgery $\sqrt{\text { dexamethasone }}$

We have no unit standard when to transfuse platelets, and nationally, there are no universally accepted guidelines for platelet transfusion in neonates. In a survey of United States and Canadian neonatologists, Josephson found $30 \%-45 \%$ of the neonatologists would transfuse platelets in a sick preterm infants with platelet counts of $>50 \times 10^{3} / \mu \mathrm{L}$ (5), a threshold of $<20 \times 10^{3} / \mu \mathrm{L}$ was selected by $<5 \%$; and $50 \%$ selected $50 \times 10^{3} / \mu \mathrm{L}$ as the "transfusion" trigger for extremely low-birthweight neonates despite the absence of apparent bleeding (5). More recently, Curley showed a platelet count threshold of $50 \times 10^{3} / \mu \mathrm{L}$ had a significantly higher rate of death or major bleeding than those transfused at a platelet count threshold of $25 \times 10^{3} / \mu \mathrm{L}$ (6). Their study included infants who were older, up to 34 weeks and did not include in their analysis any infants born at 23 weeks and no mention of how many were included who underwent major surgery as in our this case. The platelet count was $<50 \times 10^{3} / \mu \mathrm{L} 24$ times and $<25 \times 10^{3} / \mu \mathrm{L} 6$ times as late as DOL 145 . There were days when there as a rapid decrease in the platelet count to $26-50 \times 10^{3} / \mu \mathrm{L}$. We suspect by waiting, the platelet count would have eventually decreased to < $25 \times 10^{3} / \mu \mathrm{L}$, and the baby would have needed to be transfused. The number of transfusions may have been reduced if the lower threshold of $25 \times 10^{3} / \mu \mathrm{L}$ was followed, but in this sick, extremely premature infant, we were concerned the risk for significant bleed- 
ing complications would be too great using such a low threshold.

We don't have a clear understanding of why the platelet count would continue to fall to $<2510^{3} / \mu \mathrm{L}$ at five months of age. We assumed the chronic thrombocytopenia resulted from prolonged gut inflammation resulting from infection or NEC. Thrombocytopenia can be categorized kinetically as hyporegenerative, consumptive, or a mixed mechanism. The great majority of neonates who receive $>20$ platelet transfusions have a consumptive or mixed mechanism (7). The mortality rate has been reported to be $50 \%$ for infants receiving $\geq 20$ platelet transfusions. Though some of this correlation may be related to the degree of illness, platelet transfusions themselves may also be responsible for the increased mortality rate. The risk of sepsis from platelet bacterial contamination increases in those receiving $>10$ transfusions (8), so other treatment options need to be explored..

In certain thrombocytopenic conditions, corticosteroids can increase platelet counts. Bourchier and Weston reported that dexamethasone increased platelet counts, which was speculated to be on the basis of reduced inflammation and diminished platelet consumption (9). In contrast, Peng did not find dexamethasone increased platelet counts (10). Dexamethasone may have contributed to the increase in the platelet count in our case. However, the IPF was low at the time dexamethasone was started, suggesting the thrombocytopenia might have a hypoproliferative component. The platelet count continued to fall for 12 days, and she still required two platelet transfusions after starting dexamethasone.

\section{"In certain problematic cases, thrombopoietic stimulators like romiplostim might be considered to reduce or eliminate platelet transfusions (3). Early in her hospital course, the rapid decrease in the platelet count suggested a consumptive process."}

In certain problematic cases, thrombopoietic stimulators like romiplostim might be considered to reduce or eliminate platelet transfusions (3). Early in her hospital course, the rapid decrease in the platelet count suggested a consumptive process. Following sepsis and possible NEC, thrombocytopenia normally resolves in 1-2 weeks, but in some, thrombocytopenia may persist for several weeks. Before initiating romiplostim, the lower IPF indicated impaired capacity to increase platelet production, suggesting hyporegenerative thrombocytopenia (11). Sepsis or NEC can result in an insufficient compensatory increase in thrombopoiesis (12), which may explain why she responded to romiplostim. We also cannot know what effect the increased number of transfusions had on bone marrow hyporegeneration linked to transfusion inhibition of endogenous thrombopoietin as in this case. However, the platelet count rise and the IPF following romiplostim may suggest a possible boost from the exogenous thrombopoietin agonist.

The starting dose in neonates is unknown. Data from the ITP Consortium of North America ICON2 found the median starting dose was $2 \mathrm{mcg} / \mathrm{kg}$ with a maximum $10 \mathrm{mg} / \mathrm{kg} / \mathrm{dose}(2)$. The only study in a neonate started with $1 \mathrm{mcg} / \mathrm{kg} / \mathrm{dose}$ and increased up to 3 $\mathrm{mcg} / \mathrm{kg} / \mathrm{dose}$. Four doses were given over a 35 day period. (4). After discussion with our hematology, co-author consultant, we elected to start with $2 \mathrm{mcg} / \mathrm{kg} \mathrm{SQ}$ and increase the dose weekly until we had a sustained result. We doubled the dose to $4 \mathrm{mcg} / \mathrm{kg}$ after the first dose since the platelet count continued to fall. After the second dose, the platelet count began to increase four days later.

Possible complications following romiplostim have been reported to be rebound thrombocytopenia, bone marrow fibrosis, and thrombocytosis (13). Non-hematopoietic effects of romiplostim have not yet been well characterized, but recent data suggests that Tpo may result in proapoptotic and differentiating-blocking effects on neuronal cells (14), so the effects on subsequent neonatal neurodevelopment are unknown. In the first pediatric studies, the most frequent non-bleeding adverse events were headache, upper respiratory tract infections, vomiting, and oropharyngeal pain (15). No thrombotic or embolic events were noted in this baby prior to transfer, and her records were not available for review after her transfer.

This anecdotal use does not constitute a cause-and-effect relationship, nor does it establish the success of this treatment. It may have been a coincidence that the platelet count increased after the romiplostim. We would consider the cautious use of romiplostim in cases of severe and persistent thrombocytopenia in an attempt to reduce the number of platelet transfusions needed to control bleeding. We hope this case may encourage the study of romiplostim in neonates to define which platelet disorders would make this an appropriate drug for use.

\section{References}

1. Nishihira H, Toyoda Y, Miyazaki H, Kigasawa H, Ohsaki E. Growth of macroscopic human megakaryocyte colonies from cord blood in culture with recombinant human thrombopoietin (c-mpl ligand) and the effects of gestational age on frequency of colonies. Br J Haematol 1996; 92:23-8

2. Neunert CE, Rose MJ. Romiplostim for the management of pediatric immune thrombocytopenia: drug development and current practice. Blood Adv 2019; 3:1907-15.

3. Sallmon H, Gutti RK, Ferrer-Marin F, Liu Z-J, Sola-Visner $M C$. Increasing platelets without transfusion: is it time to introduce novel thrombopoietic agents in neonatal care? $\mathrm{J}$ Perinatol 2010; 30:765-9.

4. Mahat U, Talati R, Kodish E. Comment on: Use of thrombopoietin receptor agonist (romiplostim) in neonatal autoimmune thrombocytopenia due to maternal immune thrombocytopenia. Pediatr Blood Cancer 2019; 66: e27706.

5. Josephson CD, Su LL, Christensen RD, Hillyer CD, Castillejo $\mathrm{MI}$, et al. Platelet transfusion practices among neonatologists in the United States and Canada:Results of a survey. Pediatrics 2009;123:278-285.

6. Curley A, Stanworth SJ, Phil D, Willoughby K, Susanna F, et al. Randomized trial of platelet transfusion thresholds in neonates. N Engl J Med 2019;380:242-251.

7. Dohner ML, Wiedmeier SE, Stoddard RA, Null Jr D, Lambert DK, Burnett J, et al. Very high users of platelet transfusions in the neonatal intensive care unit. Transfusion 2009; 48:869-72.

8. Baer VL, Lambert DK, Henry E, Snow GL, Sola-Visner MC, Christensen RD. Do platelet transfusions in the NICU ad- 
versely affect survival? Analysis of 1600 thrombocytopenic neonates in a multihospital healthcare system. J Perinatol 2007; 27:790-6.

9. Bourchier D, Weston PJ. The effect of dexamethasone upon platelets and neutrophils of preterm infants with chronic lung disease. J Paediatr Child Health 1991; 27:101-4.

10. Peng, CT, Lin HC, Lin YJ, Tsai CH, Yeh TF. Early dexamethasone therapy and blood cell count in preterm infants. Pediatrics 1999; 104:476-481.

11. MacQueen BC, Christensen RD, Henry E, Romrell AM, Pysher TJ, Bennett ST, Sola-Visner MC. The immature platelet fraction: creating neonatal reference intervals and using these to categorize neonatal thrombocytopenias. $J$ Perinatol 2017; 37:834-8.

12. Brown RE, Rimsza KM, Pastos K, Young, Saxonhouse MA, Baily $M$, et al. Effects of sepsis on neonatal thrombopoiesis. Pediatr Res 2008; 64:399-404.

13. Kuter DJ. New thrombopoietic growth factors. Blood 2007; 109:4607-4616.

14. Ehrenreich $H$, Hasselblatt M, Knerlich F, von Ahsen $N$, Jacob $S$, Sperling S, et al. A hematopoietic growth factor, thrombopoietin, has a proapoptotic role in the brain. Proc Natl Acad Sci USA 2005;102:862-867.

15. Tarantino MD, Bussel JB, Blanchette VS,Despotovic J, Bennett $C$, Raj $A$ et al. Romiplostim in children with immune thrombocytopenia: a phase 3, randomized, double-blind, placebo-controlled study. Lancet 2016;388: 45-54.

Funding Sources: No funding has been provided to support this research.

Conflicts of Interest: The authors declare no conflicts of interest.

Author Contributions: All authors contributed to the writing and the review of this paper

Written informed consent was obtained from the mother for publication of this case report

The author has no conflicts of interests to disclose.

\section{NT}

Corresponding Author

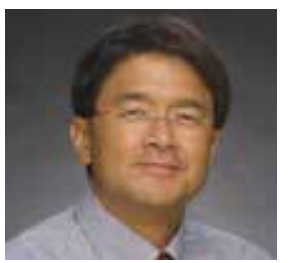

Michael Kamitsuka, MD

Division of Neonatology

Swedish Medical Center

747 Broadway, Seattle, WA 98122

Office 206-386-2159; Fax 206-386-2849

Email: Michael Kamitsuka@mednax.com

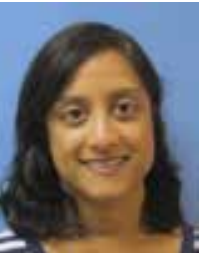

Shrena Patel, MD

Division of Neonatology

Swedish Medical Center

747 Broadway, Seattle, WA 98122

Office 206-386-2159; Fax 206-386-2849
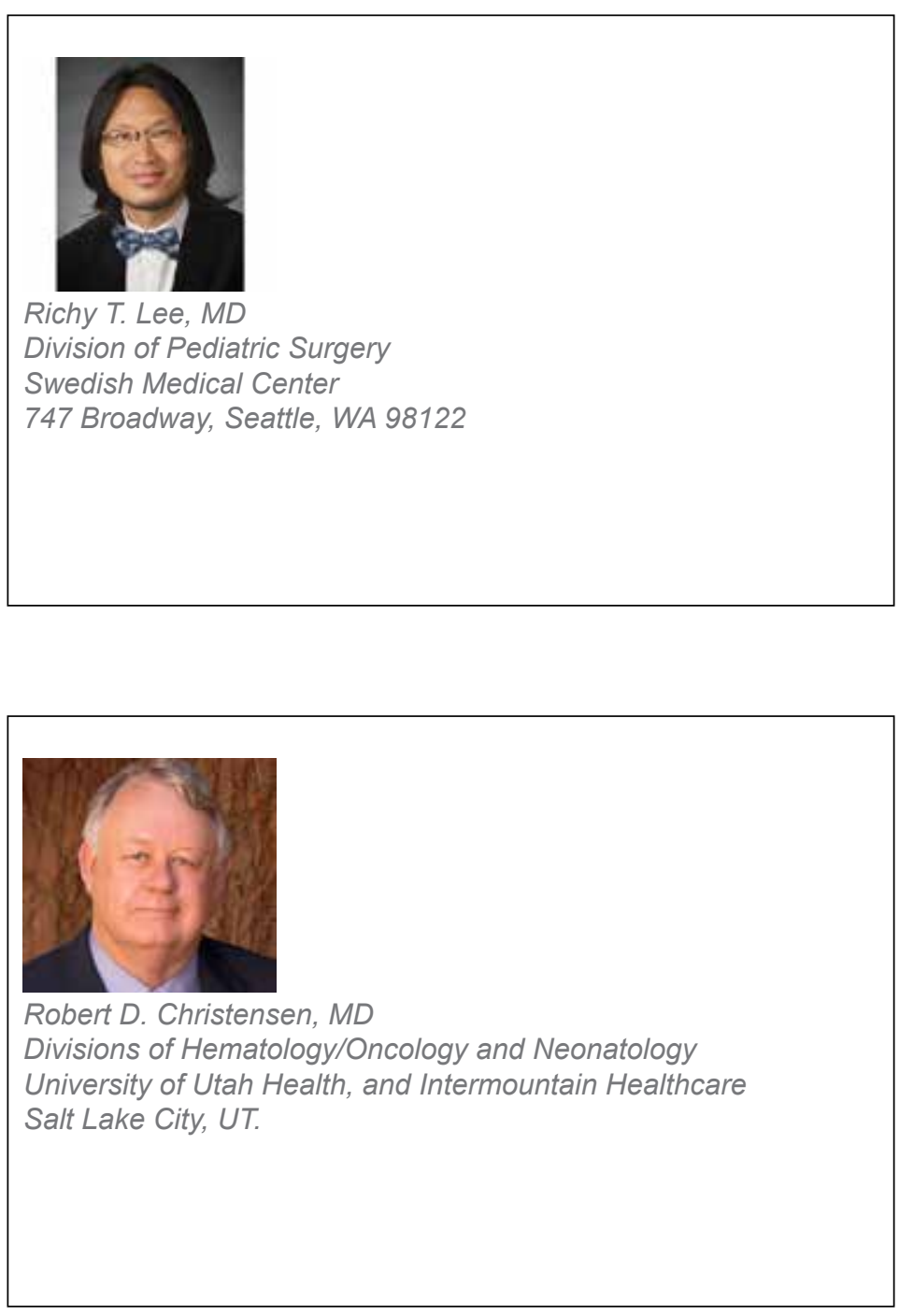